\title{
Robust model referenced control for vehicle rollover prevention with time-varying speed
}

\section{Ke Shao*}

Tsinghua Shenzhen International Graduate School,

Tsinghua University,

Shenzhen, Guangdong, 518055, China

Email: shao.ke@sz.tsinghua.edu.cn

${ }^{\star}$ Corresponding author

\section{Jinchuan Zheng}

School of Software and Electrical Engineering, Swinburne University of Technology,

Melbourne, VIC, 3122, Australia

Email: jzheng@swin.edu.au

\section{Kang Huang and Mingming Qiu}

School of Mechanical Engineering, Hefei University of Technology, Hefei, Anhui, 230001, China

Email: hfhuang98@163.com

Email: hfutqmm@hfut.edu.cn

\section{Zhe Sun}

College of Information Engineering, Zhejiang University of Technology, Hangzhou, Zhejiang, 310023, China Email: sunzhe726@zjut.edu.cn

\begin{abstract}
In this paper, an active steering control strategy is proposed to prevent rollover of a vehicle with a time-varying forward speed. The controller is designed with the aim of reducing the rollover index (RI) from an initial dangerous status to an absolutely safe status by active steering. The controller consists of two parts. The nominal control is firstly designed on the basis of the fundamental equation of constrained motion (FECM) of the vehicle, which will guarantee the nominal vehicle to track the desired states and thus rollover is prevented. To handle system uncertainties, compensatory sliding mode control (SMC) is proposed, by which the actual states are forced to track the desired ones. In the controller, the chattering problem can be alleviated by selecting a suitable performance function. To indicate the merits of the designed controller, simulation is conducted. Simulation results demonstrate that compared to conventional
\end{abstract}


controllers, the proposed controller can prevent the vehicle from rollover with stronger robustness and without system chattering.

Keywords: rollover prevention; active steering; time-varying speed; FECM; fundamental equation of constrained motion; SMC; sliding mode control; robustness.

Reference to this paper should be made as follows: Shao, K., Zheng, J., Huang, K., Qiu, M. and Sun, Z. (2021) 'Robust model referenced control for vehicle rollover prevention with time-varying speed', Int. J. Vehicle Design, Vol. 85, No. 1, pp.48-68.

Biographical notes: Ke Shao received his $\mathrm{BE}$ and $\mathrm{PhD}$ degrees in School of Mechanical Engineering from Hefei University of Technology, Hefei, China, in 2013 and 2019, respectively. From 2017 to 2019, he was a Visiting Scholar in School of Software and Electrical Engineering, Swinburne University of Technology, Melbourne, Australia, founded by China Scholarship Council. Currently, he is the Postdoc Research Fellow in Tsinghua Shenzhen International Graduate School, Tsinghua University, Shenzhen, China. His research interests include mechanical system dynamics, sliding mode control (SMC), advanced robots, high-precision motion control, and vehicle dynamics and control.

Jinchuan Zheng received his BE and ME in Mechatronics Engineering from Shanghai Jiao Tong University, Shanghai, China, in 1999 and 2002, respectively, and the $\mathrm{PhD}$ in Electrical and Electronic Engineering from Nanyang Technological University, Singapore, in 2006. In 2005, he joined the Australian Research Council Centre of Excellence for Complex Dynamic Systems and Control, School of Electrical and Computer Engineering, University of Newcastle, Newcastle, Australia, as a Research Academic. From 2011 to 2012, he worked as a Staff Engineer at Western Digital Hard Disk Drive R\&D Center, Singapore. $\mathrm{He}$ is currently an Associate Professor at Swinburne University of Technology, Melbourne, Australia. His research interests include mechanism design and control of high precision mechatronic systems, sensing and vibration analysis, dual-stage actuation, and vision-based control.

Kang Huang received his $\mathrm{PhD}$ in School of Mechanical Engineering from Hefei University of Technology, Hefei, China, in 2002. From 2006 to 2007, he was a Visiting Scholar with the University of Stuttgart, Stuttgart, Germany. $\mathrm{He}$ is currently a Professor with the School of Mechanical Engineering, Hefei University of Technology, Hefei, China. His research interests include mechanical transmission, magnetic machine, vehicles, digital design and manufacturing, information systems, robotics, dynamics, and control.

Mingming Qiu received his $\mathrm{PhD}$ in Mechatronic Engineering from Hefei University of Technology, Hefei, China, in 2015. He is currently an Assistant Professor with Hefei University of Technology. His research interests include nonlinear dynamics, complex electromechanical systems, robust control, slidingmode control, and fuzzy engineering.

Zhe Sun received his BE in Transportation Engineering from Nanjing Agricultural University, Nanjing, China, in 2011, and the PhD from Swinburne University of Technology, Melbourne, Australia, in 2017. From 2017 to 2019, he was a postdoctoral research associate in Swinburne University of Technology, 
Melbourne, Australia. Currently, he is serving as an Associate Professor in Zhejiang University of Technology, Hangzhou, China. His research interests include nonlinear control, vehicle dynamics and control, mechatronic systems and vision-based control.

\section{Introduction}

Although not occurring so often as common traffic accidents, a high level of severe injuries and deaths is caused by vehicle rollover every year (Larish et al., 2013). In general, when an emergent rollover is realised, it is often too late for a driver to respond because the vehicle handling stability will hugely fail compared to normal driving. For this reason, it is essential to develop an active assisting system to help the driver to predict a rollover risk or avoid rollover automatically.

The way of rollover prevention is essentially to reduce the load transfer between left and right wheels. Many researchers have proposed different controllers based on active steering, differential braking, anti-rollover suspension or a combination of them (Rajamani, 2011; Odenthal et al., 1999; Carlson and Gerdes, 2003; Gáspár et al., 2005; Chen and Peng, 2001; Chen et al., 2011; Sampson and Cebon, 2003b,a; Huang et al., 2012; Huang, 2009; Yim, 2011). As one of the three main effective measures, active steering will improve the vehicle states and further avoid rollover in a smoother way. Based on the steering command of the driver, the front wheel steering angle can be flexibly modified by a steering actuator (Dal Poggetto and Serpa, 2016; Beal and Gerdes, 2013; Yih and Gerdes, 2005). In Rajamani's work, electronic stability system (ESC) is utilised and countersteering by steer-by-wire (SbW) system is developed to prevent a vehicle from rollover (Rajamani and Piyabongkarn, 2013). Based on active steering, Ackermann and Gáspár solved the problem of rollover risk reduction for heavy vehicles by scheduled gains with a changing forward speed (Ackermann and Odenthal, 1999; Gáspár and Bokor, 2006). Solmaz designed a robust rollover prevention controller based on linear matrix inequality (LMI) approach, by which the output of the uncertain system is held under a certain value (Solmaz et al., 2007a,b). Imine limited the lateral acceleration to prevent heavy vehicle from rollover by using supertwisting sliding mode method (Imine et al., 2012). In most of the existing work, the controller is often designed on account of a linear parameter invariant (LPI) model which assumes a frozen forward speed. However, in practice, the vehicle speed is an important parameter of the motion equation which may largely vary and then impact the vehicle dynamics (Hegazy and Sandu, 2014). Although gain-schedule or LMI technique can play a role in solving this problem, they are difficult to design and not an analytical solution.

Udwadia-Kalaba fundamental equation of constrained motion (FECM) is firstly used in multi-body mechanical modelling problem (Udwadia and Kalaba, 2007). Based on the equation, Udwadia and Chen proposed servo constraint control method where the desired trajectory is treated as a constraint of the system (Udwadia, 2003; Chen, 2005). By transforming the trajectory constraint into a second-order form and then model it in the FECM, the control satisfying this trajectory will be designed (Chen, 1998). The benefit of this method is that it can handle linear parameter-varying (LPV) system and the control input is in an analytical form. Vehicle is a complex system with many uncertainties. Motivated by 
the variable structure control methodology, SMC is widely used in vehicle dynamics control due to its strong robustness to deal with bounded system nonlinearities and disturbances (Zheng et al., 2015). However, the switching control term in a conventional SMC controller often leads to severe system chattering problems which may defect the control performance or damage the mechanical structure (Slotine and Li, 1991).

In this paper, a robust model referenced active steering controller for rollover avoidance of a vehicle is proposed. The FECM of the vehicle is introduced to obtain the nominal control. Based on a simplified LPV vehicle model, the FECM will produce an analytical nominal steering input where the vehicle speed is allowed to vary. To overcome system uncertainties, SMC is utilised, in which a performance function is proposed for design flexibility and alleviating system chattering. Since the tyre force nonlinearity is one of the main sources of system uncertainty, to obtain the tyre force at a large slip angle region, the Magic Formula tyre model is introduced in simulation. Simulation results verify that compared to conventional SMC and PID controllers, the proposed FECM referenced controller shows stronger robustness for rollover prevention under parametric uncertainties and time-varying speed without chattering by choosing suitable control parameters.

The framework of this paper is given as follows: A simplified vehicle is modelled in Section 2 where the nonlinearity of the tyre force with respect to the slip angle is considered. Based on the roll motion of the vehicle, Section 3 quantifies the load transfer ratio (LTR) and uses it as the system output to quantify the rollover index (RI). Section 4 describes the details of the robust control design for rollover avoidance followed by a stability proof of the closed-loop system. To indicate the advantages of the proposed controller, simulation is conducted in Section 5. Finally, this paper is concluded in Section 6.

\section{Vehicle system modelling}

A bicycle model with a time-varying speed is introduced in this section to describe the vehicle dynamics. Since the tyre force is nonlinearly related to the slip angle and is one of the main sources of modelling uncertainties, to consider the tyre's impact, a nonlinear Magic Formula tyre model is also introduced. The symbols and their definitions of the used vehicle parameters in this paper are listed in Table 1, in which the subscripts $i$ indicates $f$ (front) or $r$ (rear) and $j$ indicates $l$ (left) or $r$ (right).

\subsection{A vehicle model}

A vehicle is a complex mechanical system with many degrees of freedom. For simplification, suppose that slipping does not occur at the tyres and neglect the roll motion of sprung mass as well as the effects of aerodynamics. As shown in Figure 1, the vehicle can be described by a bicycle model. For the model in Figure 1, the impact of the longitudinal tyre force on the lateral and yaw motions is ignored based on the small front wheel steering angle assumption. According to Newton's Law, the motion of the vehicle is formulated as follows (Rajamani, 2011):

$$
\begin{aligned}
m\left(\ddot{y}+V_{x} \dot{\psi}\right) & =F_{y, f}+F_{y, r}, \\
I_{z} \ddot{\psi} & =l_{f} F_{y, f}-l_{r} F_{y, r},
\end{aligned}
$$


where $y$ is the vehicle lateral displacement, $\psi$ is the vehicle heading angle. Combining equations (1) and (2) and applying small slip angle approximation, the motion equation of the vehicle can be described in a matrix form given by

$$
M \ddot{x}=A\left(V_{x}\right) \dot{x}+J \delta,
$$

where the state variable $x=\left[\begin{array}{ll}y & \psi\end{array}\right]^{T}$,

$$
\begin{aligned}
M & =\left[\begin{array}{cc}
m & 0 \\
0 & I_{z}
\end{array}\right], \\
A\left(V_{x}\right) & =\left[\begin{array}{cc}
-\frac{2 C_{\alpha, f}+2 C_{\alpha, r}}{V_{x}} & -\frac{2 C_{\alpha, f} l_{f}-2 C_{\alpha, r} l_{r}}{V_{x}}-m V_{x} \\
-\frac{2 C_{\alpha, f} l_{f}-2 C_{\alpha, r} l_{r}}{V_{x}} & -\frac{2 C_{\alpha, f}^{2} l_{f}^{2}+2 C_{\alpha, r} l_{r}^{2}}{V_{x}}
\end{array}\right], \\
J & =\left[\begin{array}{c}
2 C_{\alpha, f} \\
2 C_{\alpha, f} l_{f}
\end{array}\right] .
\end{aligned}
$$

Note that equation (3) is modelled by assuming small slip angles such that the tyre's lateral force can be approximately proportional to its slip angle, i.e., $F_{y, f}=2 C_{\alpha, f} \alpha_{f}$ and $F_{y, r}=$ $2 C_{\alpha, r} \alpha_{r}$. As shown in Figure 2, the slip angle $\alpha$ represents the angle between the heading direction and the total speed $v$ of the tyre. The slip angles of the front and rear tyres can be, respectively, given by

$$
\begin{aligned}
& \alpha_{f}=\delta-\frac{\dot{y}+l_{f} \dot{\psi}}{V_{x}}, \\
& \alpha_{r}=-\frac{\dot{y}-l_{r} \dot{\psi}}{V_{x}} .
\end{aligned}
$$

Table 1 Notations and model parameters

\begin{tabular}{ll}
\hline Parameter & Description \\
\hline$\delta$ & Steering angle of front wheel \\
$\delta_{N}$ & Nominal control input \\
$\delta_{U}$ & Compensatory control input \\
$\delta_{d}$ & Driver's steering command \\
$a_{y}$ & Vehicle lateral acceleration \\
$C_{\alpha, i}$ & Cornering stiffness of tyres \\
$\psi$ & Heading angle of vehicle \\
$V_{x}$ & Vehicle forward speed \\
$y$ & Vehicle lateral displacement \\
$F_{z, j}$ & Vertical force on tyres \\
$\alpha_{i}$ & Slip angle at tyres \\
$F_{y, i}$ & Lateral force on tyres \\
$l_{i}$ & Distance from axle to mass center \\
$g$ & Gravity acceleration \\
$I_{z}$ & Vehicle yaw moment of inertia \\
$m$ & Vehicle mass \\
$d$ & Track width \\
$h$ & Vehicle mass center height \\
\hline
\end{tabular}


Remark 1: Equation (3) is a linear parameter-varying (LPV) model with a time-varying speed which may be changed due to driver's command. Due to modelling error, some parameters in system matrices $M, A\left(V_{x}\right)$ and $J$ in equation (3) are uncertain. For instance, the mass, the moment of inertia may vary under different loads and the cornering stiffness may vary at large slip angles.

Figure 1 Bicycle model (see online version for colours)

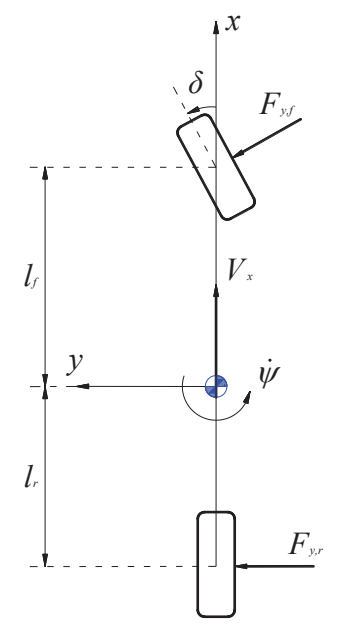

Figure 2 Slip angle at a tyre

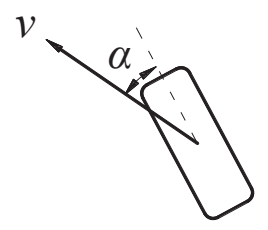

\subsection{Magic Formula tyre model}

As aforementioned, to obtain model (3), the lateral tyre force has been assumed to proportionally change as the small slip angle varies. However, due to the tyre saturation property, the nonlinear dependence of the lateral tyre force on the slip angle will be enhanced hugely at high lateral acceleration (Du et al., 2010). To consider the impact of the tyre force's nonlinear variation with the slip angle, Magic Formula tyre is introduced to model the lateral tyre force given by (Zhou, 2009)

$$
F_{y}=F_{P} \sin \left(C \arctan \left(\frac{s_{c}}{C}(1-E)+E \arctan \frac{s_{c}}{C}\right)\right) \frac{\tan \alpha}{\left\|s_{n}\right\|},
$$

where

$$
F_{P}=\frac{1.0527 F_{z} D}{1+\left(\frac{1.5 F_{z}}{m g}\right)^{3}},
$$




$$
\begin{aligned}
s_{c} & =\frac{C_{\alpha}}{F_{P}}\left\|s_{n}\right\|, \\
C_{\alpha} & =\frac{B C D}{4\left(1-e^{-\frac{c}{4}}\right)} m g\left(1-e^{-\frac{c F_{z}}{m g}}\right), \\
\left\|s_{n}\right\| & =\sqrt{\lambda^{2}+\tan ^{2} \alpha}
\end{aligned}
$$

with $F_{z}$ the tyre vertical force, $B, C, D, E$ and $c$ the parameters determined by the tyre characteristic and road condition. For a dry ground, the parameters are listed in Table 2.

Table 2 Tyre parameters under dry road condition

\begin{tabular}{ll}
\hline Symbol & Value \\
\hline$B$ & 7.15 \\
$C$ & 2.30 \\
$D$ & 0.87 \\
$E$ & 1.00 \\
$c$ & 1.54 \\
\hline
\end{tabular}

And $\lambda$ is the slip ratio defined by

$$
\lambda= \begin{cases}\frac{r_{e f f} \omega_{w}-v_{w}}{v_{w}}, & r_{e f f} \omega_{w}<v_{w} \\ \frac{r_{e f f} \omega_{w}-v_{w}}{r_{e f f} \omega_{w}}, & r_{e f f} \omega_{w} \geq v_{w}\end{cases}
$$

where $r_{\text {eff }} \omega_{w}$ is the circumferential speed of wheel, $v_{w}$ is the hub speed, and $\alpha$ is the slip angle which has been given by equations (4) and (5).

It is clear from equation (6) that the lateral force is impacted by the levels of vertical force of the tyre, slip angle and slip ratio. For the front tyre of the vehicle (3) with the parameters as listed in Table 3, assuming the vehicle speed $V_{x}=120 \mathrm{~km} / \mathrm{h}$, the lateral tyre force variation with the slip angle at different slip ratio levels of the front wheel is shown in Figure 3. We can from Figure 3 that at large slip angles, the nonlinear dependence of the tyre force on the slip angle is enhanced hugely.

Table 3 Parameters of an SUV

\begin{tabular}{lc}
\hline Symbol & Value \\
\hline$m$ & $1987.935 \mathrm{~kg}$ \\
$I_{z}$ & $2488 \mathrm{kgm}^{2}$ \\
$g$ & $9.8 \mathrm{~m} / \mathrm{s}^{2}$ \\
$d$ & $1.48 \mathrm{~m}$ \\
$l_{f}$ & $1.1473 \mathrm{~m}$ \\
$l_{r}$ & $1.1307 \mathrm{~m}$ \\
$h$ & $0.306 \mathrm{~m}$ \\
\hline
\end{tabular}


Figure 3 Lateral tyre force variations at different slip ratio levels (see online version for colours)

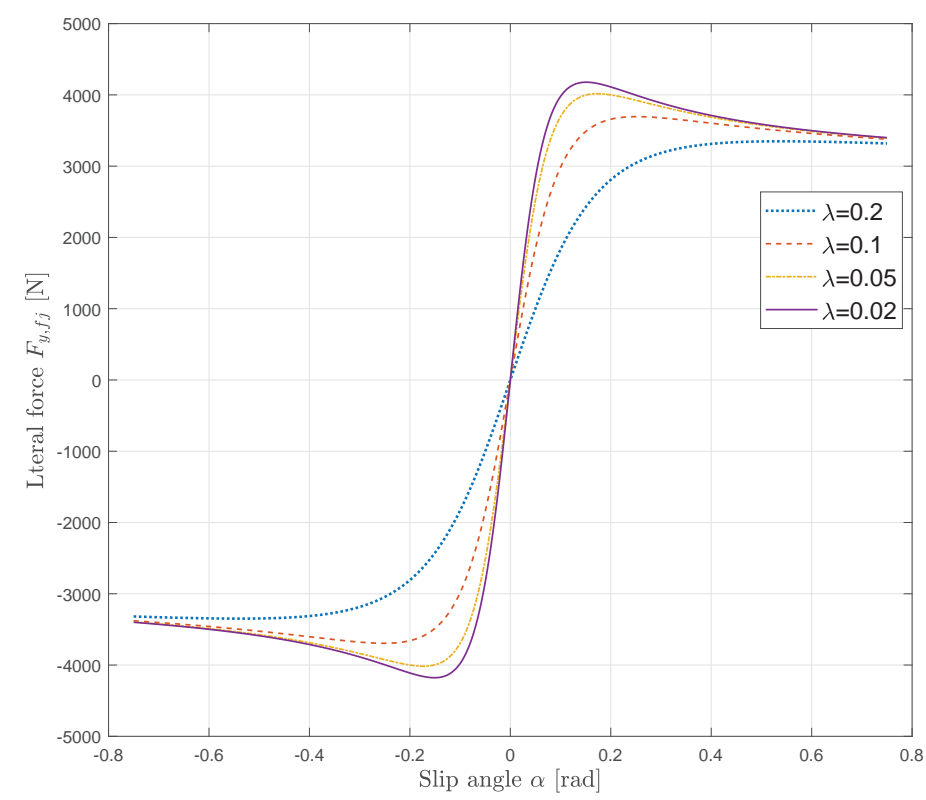

\section{Load transfer ratio}

Figure 4 describes the roll motion of a vehicle. As shown in Figure 4, the vertical loads between the left and right wheels transfer due to the impact of lateral acceleration $a_{y}$. The LTR is used as the rollover index (RI) in our research (Shao et al., 2019). LTR is constructed before the inner wheel lifts off the ground and it can be given by

$$
\mathrm{LTR}=\frac{F_{z, r}-F_{z, l}}{F_{z, r}+F_{z, l}},
$$

where $F_{z, r}$ is the left wheel vertical load and $F_{z, l}$ denotes the right wheel vertical force as shown in Figure 4. Due to $F_{z, r}+F_{z, l}=m g$, it is obvious that $-1 \leq \mathrm{LTR} \leq 1$. Specifically, $\mid$ LTR $\mid<1$ indicates a safe cornering condition. When the inner wheel lifts off the ground, $|\mathrm{LTR}|=1$, i.e., the vehicle rolls over as defined in this paper. If the LTR value is closer to zero, the safer condition can be guaranteed.

To quantify LTR, assume that the roll center of the vehicle locates at the middle of the track. As shown in Figure 4, considering the torque balance along the roll center, LTR is given by Rajamani (2011)

$$
\mathrm{LTR}=\frac{2 h}{d g} a_{y} .
$$

Generally, since the active rollover prevention should be actuated before a rollover is detected, an LTR threshold is often chosen for prediction (Ackermann and Odenthal, 1999). Once LTR reaches the threshold the controller will permit the LTR to continue to grow such that the rollover will be avoided. In this research, to verify the effectiveness of the controller, our control objective is to reduce $|\mathrm{LTR}|$ from 1 to 0 , i.e., from the status when 
the lateral acceleration is $\left|a_{y}\right|=23.72 \mathrm{~m} / \mathrm{s}^{2}$ which can be calculated from equation (13), to an absolutely safe status $\left(a_{y}=0\right)$.

Figure 4 Roll motion of vehicle (see online version for colours)

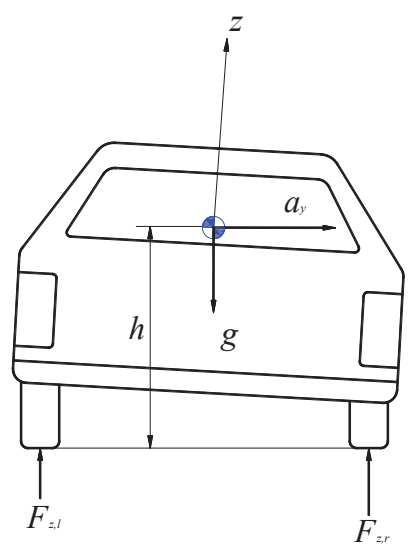

\section{Active rollover prevention control}

In this section, we design an active steering controller for the robust rollover prevention on the basis of model (3). The control objective is to adjust the vehicle from a rollover boundary $(|\mathrm{LTR}|=1$ ) to an absolutely safe status. A nominal control component is first produced based on FECM and then a compensatory SMC component is further developed to deal with system uncertainties.

\subsection{FECM referenced chattering free controller}

The fundamental equation of constrained motion (FECM) is firstly introduced in Udwadia and Kalaba's publications and is used to produce the servo control input for a desired trajectory (Udwadia and Kalaba, 2007). The control based on FECM is an analytical control scheme and is easy to design. Firstly, according to Newton's law, without consideration of the desired trajectory and the servo inputs, a mechanical system can be modelled by the following equation:

$$
M(x, t) \ddot{x}=F^{U}(x, \dot{x}, t),
$$

where $M$ is the system inertia matrix, the force vector $F^{U}$ can contain gravitational force, centrifugal force and other control inputs. Secondly, the desired tracking trajectory is treated as a constraint in this method and can be described in a second-order equation given by

$$
G(x, t) \ddot{x}=b(x, \dot{x}, t),
$$

where $G$ is the constraint matrix. Finally, to track the desired trajectory, a servo control input $F^{C}$ must be added onto the unconstrained model (14). The closed-loop system can thus be described by

$$
M(x, t) \ddot{x}=F^{U}(x, \dot{x}, t)+F^{C}(x, \dot{x}, t),
$$


where $F^{C}$ is the servo control input to guarantee the constraint (15). Equation (17) is called the FECM. Udwadia and Kalaba verified that $F^{C}$ can be formulated in the form of:

$$
\begin{aligned}
F^{C}(x, \dot{x}, t)= & M(x, t)^{\frac{1}{2}}\left(G(x, t) M(x, t)^{-\frac{1}{2}}\right)^{+}(b(x, \dot{x}, t) \\
& \left.-G(x, t) M(x, t)^{-1} F^{U}(x, \dot{x}, t)\right),
\end{aligned}
$$

where the symbol "+" denotes Moore-Penrose pseudoinverse.

It has been verified that by choosing a suitable Lyapunov function the control input (17) will guarantee system (16) asymptotically stable even though the initial condition does not satisfy the constraint (15). The proof details can be seen in Udwadia (2003).

Next, the FECM is utilised for the vehicle rollover prevention in this research. Recalling equation (13), the control objective, i.e., the desired trajectory, is described by

$$
\begin{aligned}
a_{y} & =\ddot{y}+V_{x} \dot{\psi} \\
& =0,
\end{aligned}
$$

Furthermore, note that the system (3) has two states but only one input. By eliminating the input in equation (3), an additional modelling constraint can be obtained (Koganti and Udwadia, 2016)

$$
m l_{f} \ddot{y}-I_{z} \ddot{\psi}=-2 \frac{C_{\alpha, r}\left(l_{f}+l_{r}\right)}{V_{x}} \dot{y}+\left(2 \frac{C_{\alpha, r} l_{r}\left(l_{f}+l_{r}\right)}{V_{x}}-m l_{f} V_{x}\right) \dot{\psi} .
$$

Combining equations (18) and (19), the system constraint can be rewritten in the form (15) where

$$
\begin{aligned}
G & =\left[\begin{array}{cc}
1 & 0 \\
m l_{f}-I_{z}
\end{array}\right], \\
b\left(V_{x}, \dot{x}\right) & =\left[\begin{array}{cc}
0 & -V_{x} \\
-2 \frac{C_{\alpha, r}\left(l_{f}+l_{r}\right)}{V_{x}} & 2 \frac{C_{\alpha, r} l_{r}\left(l_{f}+l_{r}\right)}{V_{x}}-m l_{f} V_{x}
\end{array}\right] \dot{x} .
\end{aligned}
$$

Considering the vehicle model (3), it is obvious that $F^{U}=A\left(V_{x}\right) \dot{x}$ and $F^{C}=J \delta$. From equation (17), the nominal steering input to force the nominal system (3) to track the desired trajectory (18) can be given by

$$
\delta_{N}=J^{+} M^{\frac{1}{2}}\left(G M^{-\frac{1}{2}}\right)^{+}\left(b\left(V_{x}, \dot{x}\right)-G M^{-1} A\left(V_{x}\right) \dot{x}\right) .
$$

Remark 2: For the nominal vehicle (3), once the steering input (20) is applied, the constraint (18) will be guaranteed, i.e., rollover is prevented. The control based on FECM is suitable for an LPV vehicle system and it is in an analytical form. As shown in equation (20), matrices $A\left(V_{x}\right)$ and $b\left(V_{x}, \dot{x}\right)$ are both time-varying. To illustrate this method, the vehicle speed is set to be time-varying in this paper.

The simplified model (3) ignores some uncertainties of the vehicle such as the nonlinearly related lateral tyre force and slip angle, the variable mass and inertia of moment of the vehicle. Therefore, the actual trajectory will not track the desired trajectory (18) when only the nominal control input (3) is applied (Udwadia and Wanichanon, 2014). To deal with 
system uncertainties, a compensatory steering input must be added for robust performance as required in a practical vehicle. Equation (3) is thus extended as

$$
M_{a} \ddot{x}_{c}=A_{a}\left(V_{x}\right) \dot{x}_{c}+J_{a} \delta_{N}+J_{a} \delta_{U},
$$

where $\delta_{U}$ is the compensatory control input to be designed, the subscript " $a$ " denotes the actual system, $x_{c}=\left[y_{c} \psi_{c}\right]^{T}$ is the closed-loop system state and $\delta_{N}$ is the nominal steering angle formulated in (20) to control the nominal system (3) to satisfy the constraint (18). The control objective is that the actual system state $x_{c}$ is guaranteed to track the nominal system state $x$ and thus the constraint (18) is fulfilled. To solve $\delta_{U}$, define the error vector as

$$
e=x_{c}-x,
$$

where $x \in \mathrm{R}^{2 \times 1}$ is the state of the nominal system (3) under the nominal control input (20). Combining Equations (3) and (21), we have

$$
\begin{aligned}
\ddot{e} & =\ddot{x}_{c}-\ddot{x} \\
& =M_{a}^{-1}\left(A_{a}\left(V_{x}\right) \dot{x}_{c}+J_{a} \delta_{N}\right)-M^{-1}\left(A\left(V_{x}\right) \dot{x}+J \delta_{N}\right)+M_{a}^{-1} J_{a} \delta_{U} .
\end{aligned}
$$

Note that in equation (23), the coefficient of steering input $\delta_{U}$, i.e., $M_{a}^{-1} J_{a}$, contains system uncertainties. For the control design, we assume

$$
M_{a}^{-1} J_{a} \delta_{U}=M^{-1} J \delta_{U}+\xi
$$

where $\xi$ denotes the resulting uncertainties due to assumption. Define $\Delta \ddot{x}=$ $M_{a}^{-1}\left(A_{a}\left(V_{x}\right) \dot{x}_{c}+J_{a} \delta_{N}\right)-M^{-1}\left(A\left(V_{x}\right) \dot{x}+J \delta_{N}\right) \quad$ and $\quad u=M^{-1} J \delta_{U}$. Then, equation (23) reduces to

$$
\ddot{e}=u+\xi+\Delta \ddot{x},
$$

where the term $\xi+\Delta \ddot{x}$ is assumed to be bounded by

$$
\|\xi+\Delta \ddot{x}\| \leq \bar{\xi}
$$

Note that $u$ will be firstly designed before the compensatory control input $\delta_{U}$ is obtained. Define the generalised sliding mode surface $s \in \mathrm{R}^{2 \times 1}$ given by Edwards and Spurgeon (1998)

$$
s=\dot{e}+\lambda_{1} e+\lambda_{2} \int e \mathrm{~d} t,
$$

where $\lambda_{1}>0, \lambda_{2} \geq 0$ are the control parameters to be selected. The SMC input $u$ is designed as follows:

$$
u=-\lambda_{1} \dot{e}-\lambda_{2} e-\frac{\bar{\xi}}{\epsilon} s-f(s)
$$

where $\epsilon$ is a small positive number denoting the sliding mode layer thickness. $f(s) \in \mathrm{R}^{2 \times 1}$ is a performance function vector for design flexibility which satisfies

$$
s^{T} f(s) \geq 0
$$


Remark 3: Conventional SMC controller may lead to system chattering due to the switching term. The chattering may not only cause damage to the mechanical structure but also defect the control performance. The control input (28) can produce no system chattering with a suitable choice of $f(s)$. For instance, one can design $f(s)$ as a polynomial vector $f(s)=\left[\begin{array}{lll}\sum_{k=0}^{K} a_{k} s_{1}^{2 k+1} & \sum_{l=0}^{L} b_{l} s_{2}^{2 l+1}\end{array}\right]^{T}$ where the non-negative parameters $K, L, a_{k}$ and $b_{l}$ can be appropriately selected for desired system performance.

To verify the control input (28), choose the Lyapunov function given by

$$
V=\frac{1}{2} s^{T} s
$$

From equation (30), for $|s|>\epsilon$, the derivative of $V$ is obtained by

$$
\begin{aligned}
\dot{V} & =s^{T} \dot{s} \\
& =s^{T}\left(\ddot{e}+\lambda_{1} \dot{e}+\lambda_{2} e\right) \\
& =s^{T}\left(u+\xi+\Delta \ddot{x}+\lambda_{1} \dot{e}+\lambda_{2} e\right) \\
& =s^{T}\left(-\lambda_{1} \dot{e}-\lambda_{2} e-\frac{\bar{\xi}}{\epsilon} s-f(s)+\xi+\Delta \ddot{x}+\lambda_{1} \dot{e}+\lambda_{2} e\right) \\
& =s^{T}\left(-\frac{\bar{\xi}}{\epsilon} s-f(s)+\xi+\Delta \ddot{x}\right) \\
& =-\frac{\bar{\xi}}{\epsilon} s^{T} s+s^{T}(\xi+\Delta \ddot{x})-s^{T} f(s) \\
& <-\frac{\bar{\xi}}{\|s\|} s^{T} s+s^{T}(\xi+\Delta \ddot{x})-s^{T} f(s) \\
& =-\|s\| \bar{\xi}+s^{T}(\xi+\Delta \ddot{x})-s^{T} f(s) \\
& \leq-\|s\| \bar{\xi}+\|s\|\|\xi+\Delta \ddot{x}\|-s^{T} f(s) \\
& =-\|s\|(\bar{\xi}-\|\xi+\Delta \ddot{x}\|)-s^{T} f(s) .
\end{aligned}
$$

Recalling equations (26) and (29), due to $\|\xi+\Delta \ddot{x}\| \leq \bar{\xi}, s^{T} f(s) \geq 0$, we have $-\|s\|(\bar{\xi}-$ $\|\xi+\Delta \ddot{x}\|)-s^{T} f(s) \leq 0$. Therefore, $\dot{V}<0$ is guaranteed. Then, from equations (18), (22) and (27), $a_{y} \rightarrow 0$ is thus ensured for all $|s|>\epsilon$. The proof is thus completed.

Given $u=M^{-1} J \delta_{U}$, from equation (28), the compensatory control input is obtained by

$$
\begin{aligned}
\delta_{U} & =J^{+} M u \\
& =J^{+} M\left(-\lambda_{1} \dot{e}-\lambda_{2} e-\frac{\bar{\xi}}{\epsilon} s-f(s)\right) .
\end{aligned}
$$

Combining equation (20) with equation (32), the total control input is then obtained by

$$
\begin{aligned}
\delta= & \delta_{N}+\delta_{U} \\
= & J^{+} M^{\frac{1}{2}}\left(G M^{-\frac{1}{2}}\right)^{+}\left(b\left(V_{x}, \dot{x}\right)-G M^{-1} A\left(V_{x}\right) \dot{x}\right) \\
& +J^{+} M\left(-\lambda_{1} \dot{e}-\lambda_{2} e-\frac{\bar{\xi}}{\epsilon} s-f(s)\right),
\end{aligned}
$$

where $\delta$ is the total steering angle, $\delta_{N}$ and $\delta_{U}$ represent the nominal control component (20) and compensatory control component (28), respectively. 


\subsection{Control implementations}

The proposed control diagram is illustrated in Figure 5. In practice, the forward speed $V_{x}$ is difficult to be measured directly. To obtain $V_{x}$, observer technique is usually used to estimate the lateral speed or slip angle information firstly (Limroth, 2009). In our research, the vehicle forward speed is assumed to be known. The yaw rate can be directly obtained from the installed anti-lock braking system (ABS) or electronic stability control (ESC) system. As a result, the vehicle lateral displacement $y$ and the orientation angle $\psi$ can be obtained by the integrations of them. The vehicle speed and lateral acceleration can also be monitored from the ABS or ESC systems. The vehicle speed is designed as time-varying in the proposed controller. Once the lateral acceleration is detected to exceed the boundary value, i.e., $a_{y}>23.72 \mathrm{~m} / \mathrm{s}^{2}$, the controller will generate a new steering command (33) to replace the driver's command to reach an absolutely safe status.

Figure 5 Diagram of the active rollover prevention control system

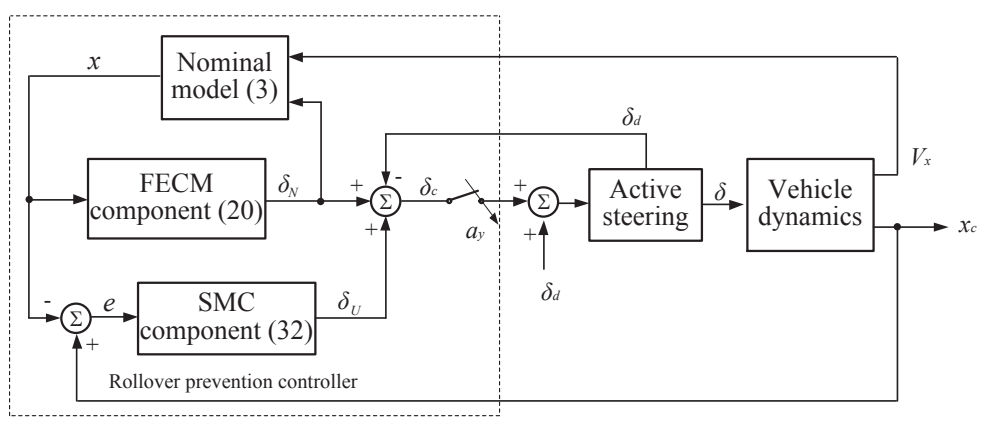

To compensate the driver's command, the actual control input of the rollover prevention controller is $\delta_{c}=\delta-\delta_{d}$, as shown in Figure 5. It is clear that at a safe status, since the controller is switched off, the steering angles of both the nominal vehicle and the actual vehicle are reduced the driver's command $\delta_{d}$ only. At an unsafe status, the rollover prevention controller is switched on and steering inputs of the nominal vehicle and the actual vehicle are replaced by $\delta_{N}$ and $\delta$, respectively. Under the nominal control input $\delta_{N}$, the nominal vehicle generates a reference state $x$ to guarantee the constraint (18) as shown in Figure 5. The SMC controller will further guarantee actual vehicle state $x_{c}$ to track $x$ and the rollover is thus prevented.

Four parameters are to be selected in the controller of (28) for the desired performance. The choices of $\lambda_{1}$ and $\lambda_{2}$ can refer to the guideline of the proportional and integral coefficients for a traditional PI controller. In this research, we choose $\lambda_{1}=10$ and $\lambda_{2}=5$. As shown in equation (26), $\bar{\xi}$ must be larger than the bound of the system uncertainties. $\epsilon$ is the sliding mode layer thickness, out of which the system will be led by the controller to the sliding surface. That is, $\epsilon$ determines the size of the neighbourhood around zero for the sliding variable to asymptotically converge and a small value of which implies a high precision but an increased amplitude of the control input in equation (28). For the vehicle in this paper, we choose $\bar{\xi}=0.01$ and $\epsilon=0.005$.

The choice of the performance function $f(s)$ will impact the system performance as well. For instance, one can choose $f(s)=\operatorname{sgn}(\mathrm{s})$, however, this may cause system chattering problem. As aforementioned, to obtain a chattering free control, a polynomial vector can 
be selected. Furthermore, we can see from equation (31) that the choice of $f(s)$ will also impact the convergence rate of the system. In this paper, we simply select $f(s)=s$.

\section{Simulation results}

To verify the proposed controller for vehicle rollover prevention, MATLAB simulations are conducted in this section. The vehicle will firstly be driven to generate a threshold lateral acceleration and then be adjusted by the controller. Nonlinear model (1)-(2) is adopted in the simulation where the Magic Formula tyre model (6) is utilised to calculate the lateral tyre force. The SUV parameters as listed in Table 3 are used in the simulation. In the control input (33), the cornering stiffness coefficients are $C_{\alpha, f}=27748 \mathrm{~N} / \mathrm{rad}$ and $C_{\alpha, r}=54700$ $\mathrm{N} / \mathrm{rad}$. To mimic a slalom path for testing, the steering command of driver $\delta_{d}$ is assumed to be a sinusoidal signal as described in Figure 6 . The forward speed is given by $V_{x}=$ $(120+5 \sin 0.2 \pi t) \mathrm{km} / \mathrm{h}$. To test the controller's robustness against parameter variation, the vehicle mass $m$ as well as its moment of inertia $I_{z}$ is increased by $10 \%$ and the roll radius $h$ is increased by $5 \%$.

Figure 6 Front wheel steering angle command for simulation (see online version for colours)

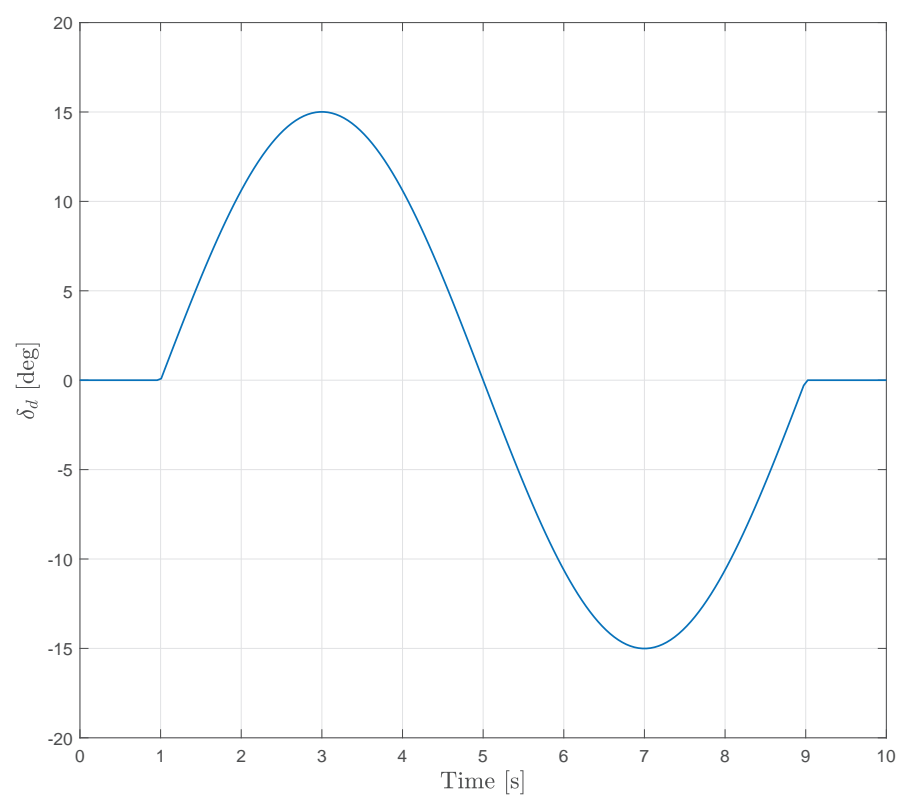

\subsection{SMC controller for comparison}

For comparison, a conventional sliding mode controller is introduced. To prevent rollover, the lateral acceleration will be reduced to 0 as aforementioned. Based on the vehicle model (3), the system output in equation (18) can be described by 


$$
\begin{aligned}
a_{y}= & -\frac{2 C_{\alpha, f}+2 C_{\alpha, r}}{m V_{x}} \dot{y}-\frac{2 C_{\alpha, f} l_{f}-2 C_{\alpha, r} l_{r}}{m V_{x}} \dot{\psi} \\
& +\frac{2 C_{\alpha, f}}{m} \delta+\xi^{\prime},
\end{aligned}
$$

where $\xi^{\prime}$ is from the system output uncertainties. Define the error $e^{\prime}=a_{y}-0=a_{y}$. Since $\frac{2 C_{\alpha, f}}{m} \delta$ is a feedthrough term of the system output (34), to remove the differential of the control input, the sliding surface is designed as an integral of the error given by Kunnappillil Madhusudhanan et al. (2015)

$$
\begin{aligned}
s & =\int e^{\prime} \mathrm{d} t \\
& =\int a_{y} \mathrm{~d} t .
\end{aligned}
$$

Design the SMC as follows:

$$
\delta_{S M C}=\frac{C_{\alpha, f}+C_{\alpha, r}}{C_{\alpha, f} V_{x}} \dot{y}+\frac{C_{\alpha, f} l_{f}-C_{\alpha, r} l_{r}}{C_{\alpha, f} V_{x}} \dot{\psi}-\frac{m}{2 C_{\alpha, f}} \lambda \operatorname{sgn}(\mathrm{s}),
$$

where $\lambda$ is selected to deal with the uncertainty $\xi^{\prime}$. In this paper, we choose $\lambda=0.0015$, and a frozen speed of $V_{x}=120 \mathrm{~km} / \mathrm{h}$ is used in the controller design of (36).

\subsection{PID controller for comparison}

A typical PID controller is also introduced for comparison, given by

$$
\delta_{P I D}=K_{P} e^{\prime}+K_{I} \int_{0}^{t} e^{\prime} \mathrm{d} \tau+K_{D} \dot{e}^{\prime}
$$

where the error $e^{\prime}$ was defined in (35). The control parameters are selected as $K_{P}=1$, $K_{I}=0.1$ and $K_{D}=0.1$.

\subsection{Simulation analysis}

The lateral accelerations of the vehicle are shown in Figure 7. We can see from Figure 7 that the lateral acceleration reaches the threshold value of $23.72 \mathrm{~m} / \mathrm{s}^{2}$ at about $2.75 \mathrm{~s}$, indicating that the uncontrolled vehicle rolls over. The vehicles under the proposed controller and the compared SMC and PID controllers can all avoid rollover successfully. However, the SMC controller consumes more time to force the lateral acceleration to zero and produces severe chattering. Compared to the SMC controller, the lateral acceleration converges to zero faster under the proposed controller and without system chattering. As for the PID controller, larger overshoot is generated. The control inputs are shown in Figure 8. It is clear that compared to both SMC and PID controllers, the proposed controller generates smaller steering input, implying less energy consumption. This is because in the FECM controller, the nominal controller generate majority of steering input and the compensatory controller will minorly compensate for the parameter uncertainties. Besides, because of the selection 
of the performance function, the control input (33) does not consist of a switching term like that in SMC controller (36). On the contrary, since the SMC controller is designed at a frozen speed, the controller has to generate more energy to tolerate the speed variation.

Figure 7 Lateral acceleration variation of the open-loop and controlled vehicle (see online version for colours)

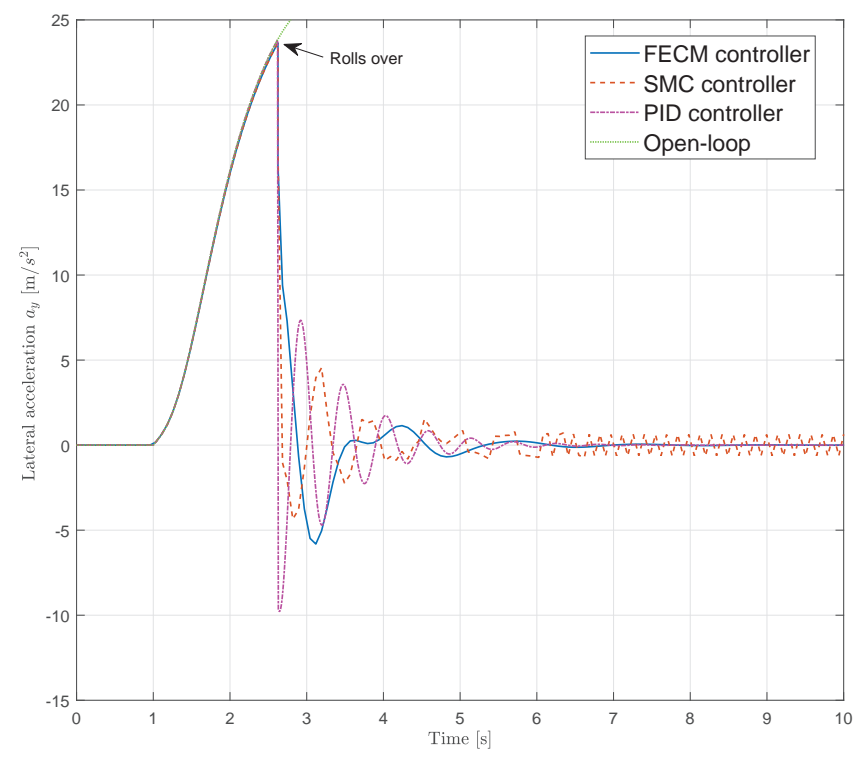

Figure 8 Control inputs of the proposed FECM controller and compared controllers (see online version for colours)

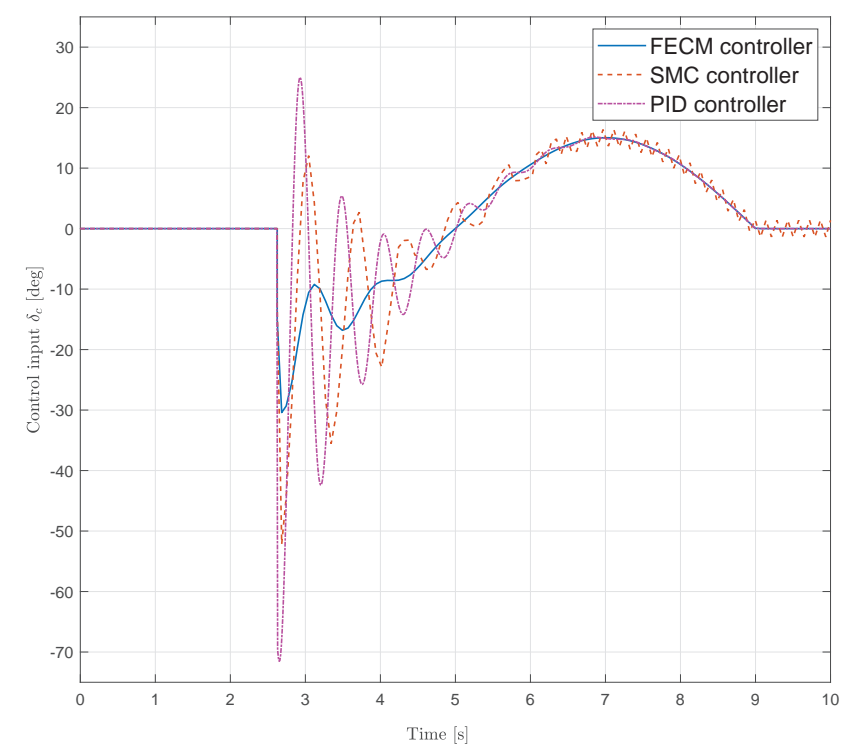


Figure 9 Vehicle state variation of the open-loop and controlled vehicles: (a) variations of vehicle lateral displacement and (b) variations of vehicle heading angle (see online version for colours)

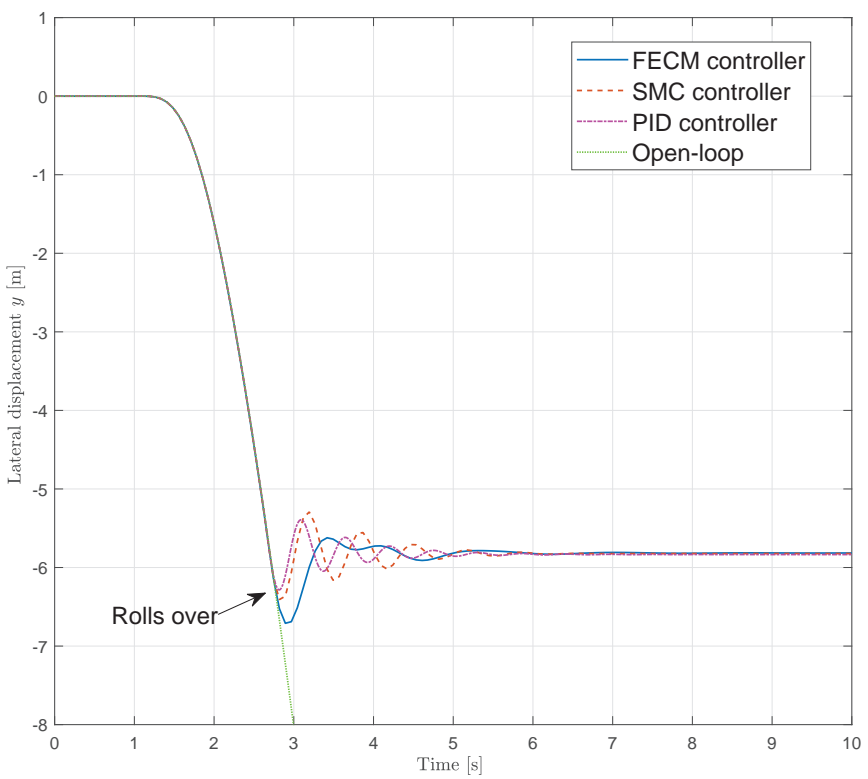

(a)

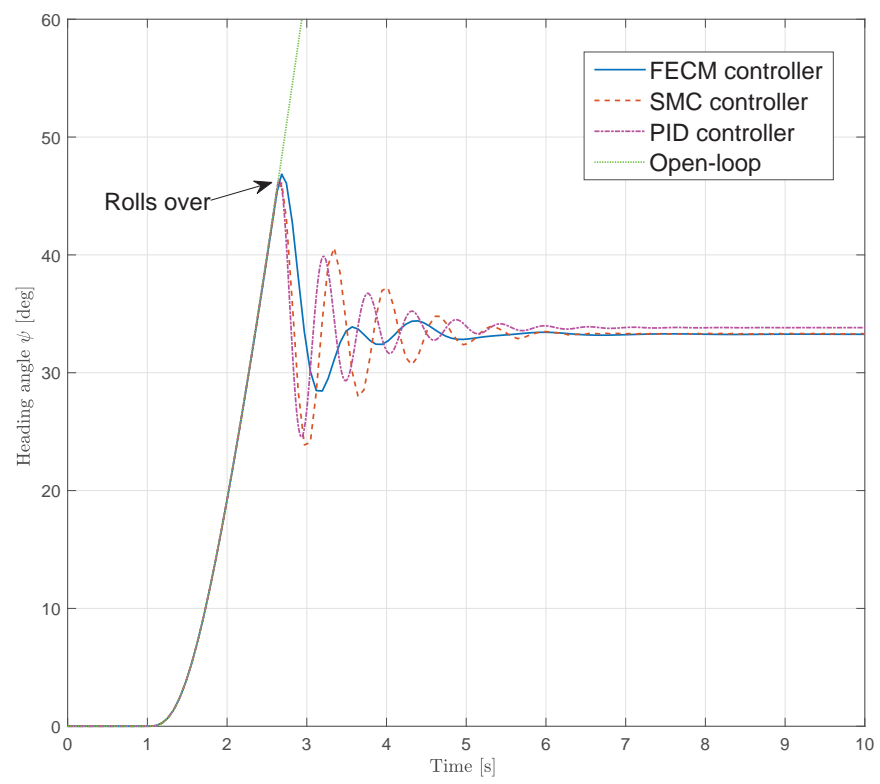

(b) 
Figure 10 Errors between the controlled and the desired $\ddot{y}$ and $\dot{\psi}$ : (a) errors of second derivative of lateral displacement and (b) errors of first derivative of heading angle (see online version for colours)

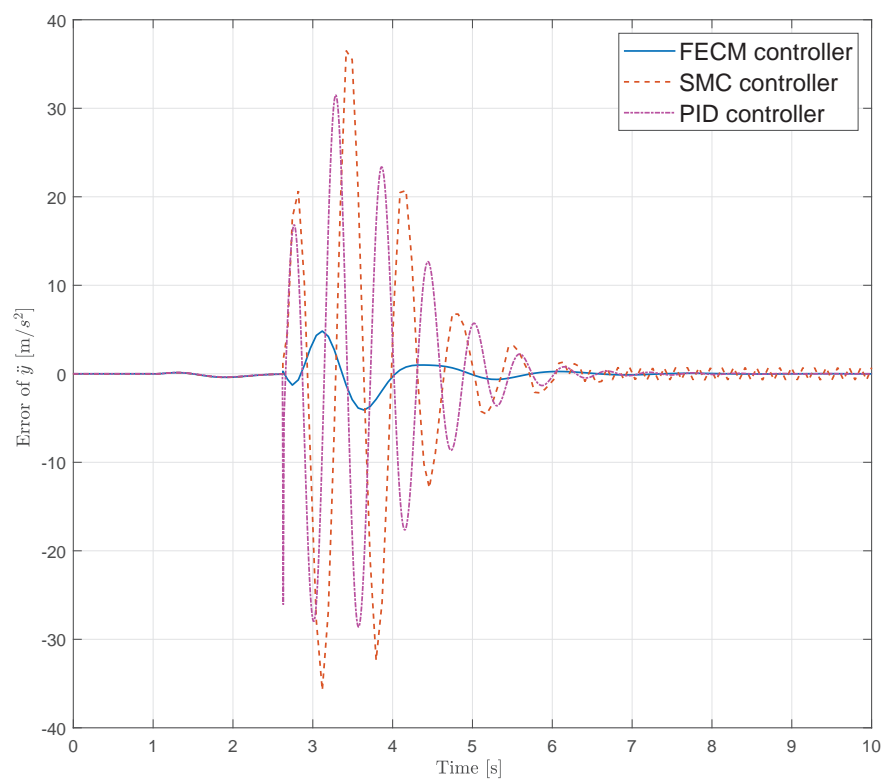

(a)

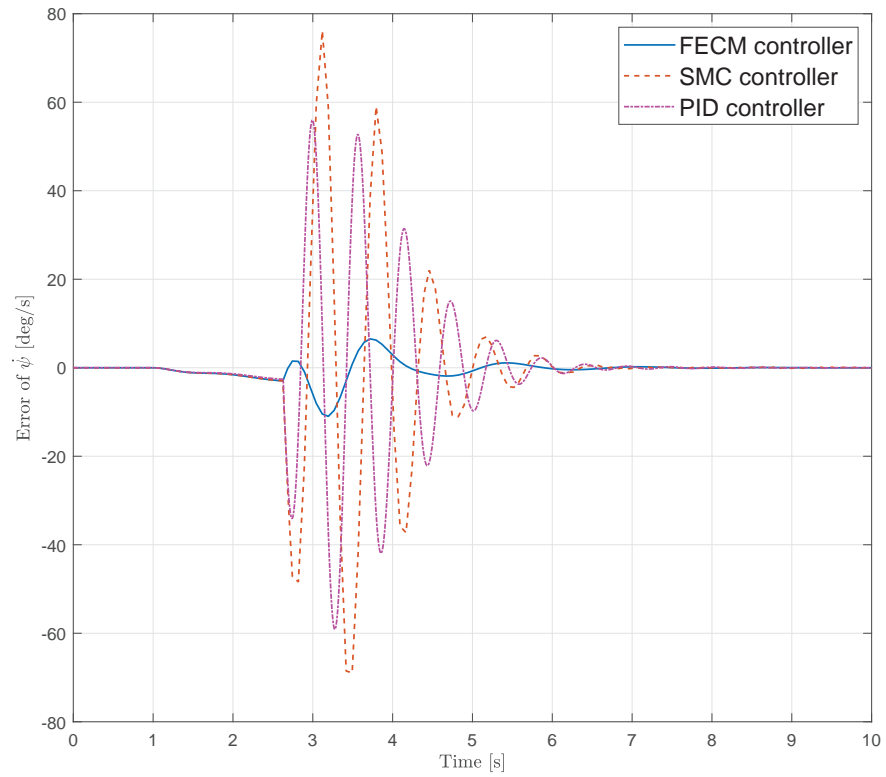

(b) 
The vehicle states variations are shown in Figure 9. It is clear that to guarantee the constraint (18) the lateral displacement and heading angle of the controlled vehicle are largely adjusted. We can see from Figure 9 that the states of the vehicle under the proposed controller converge faster than those under both SMC and PID controllers. For the proposed controller, to guarantee the constraint (18), the vehicle states $x_{c}$ are controlled to track the states $x$ of the nominal vehicle (3). The errors of $\ddot{y}$ and $\dot{\psi}$ between the controlled vehicle and the nominal vehicle are illustrated in Figure 10. We can see from Figure 10 that compared to the conventional controllers, $\ddot{y}$ and $\dot{\psi}$ under the proposed controller can well track those of the nominal vehicle with smaller errors. Note that before the rollover prevention controller is activated, the steering inputs of the nominal and actual vehicles are both the driver's command $\delta_{d}$ such that the errors between $x_{c}$ and $x$ are really small (Figure 5).

The simulation results verify that the proposed controller can prevent the vehicle from rollover with strong robustness and without chattering under system uncertainties and speed variation.

\section{Conclusion}

In this paper, vehicle rollover prevention by limiting its lateral acceleration based on active steering is studied. An LPV vehicle model is presented, which is associated with a time-varying forward speed. To prevent rollover, an FECM referenced robust controller is introduced. The FECM is suitable for the second-order LPV vehicle model with the continuously changed speed. Based on the FECM of the vehicle, an analytical nominal feedforward control is firstly developed to guarantee the linear vehicle model to track the desired states and furthermore the lateral acceleration is guaranteed to converge to the desired one. Because of the parameter uncertainties, the states of the actual vehicle will not track the nominal ones only under the nominal control. To deal with this, a compensatory SMC controller is designed where the control performance can be guaranteed and the chattering problem can be alleviated by choosing suitable control parameters. To verify the proposed controller, simulation with time-varying speed and system parameter uncertainties is studied. The simulation results demonstrate that compared to the conventional controllers, the lateral acceleration is reduced to zero faster and more smoothly without system chattering.

\section{Acknowledgement}

This project was supported by the National Key R\&D Program of China, under Grant No. 2017 YFB0103204/2017YFB0103201.

\section{References}

Ackermann, J. and Odenthal, D. (1999) 'Damping of vehicle roll dynamics by gain scheduled active steering', European Control Conference (ECC), Karlsruhe, Germany, IEEE, pp.4100-4106.

Beal, C.E. and Gerdes, J.C. (2013) 'Model predictive control for vehicle stabilization at the limits of handling', IEEE Transactions on Control Systems Technology, Vol. 21, No. 4, pp.1258-1269. 
Carlson, C.R. and Gerdes, J.C. (2003) 'Optimal rollover prevention with steer by wire and differential braking', ASME International Mechanical Engineering Congress, Washington DC, American Society of Mechanical Engineers, Washington DC, USA, pp.345-354.

Chen, B-C. and Peng, H. (2001) 'Differential-braking-based rollover prevention for sport utility vehicles with human-in-the-loop evaluations', Vehicle System Dynamics, Vol. 36, Nos. 4-5, pp.359-389.

Chen, B-C., Yu, C-C., Hsu, W-F. and Lo, M-F. (2011) 'Design of electronic stability control for rollover prevention using sliding mode control', International Journal of Vehicle Design, Vol. 56, Nos. 1-4, pp.224-245.

Chen, Y-H. (1998) 'Second-order constraints for equations of motion of constrained systems', IEEE/ASME Transactions on Mechatronics, Vol. 3, No. 3, pp.240-248.

Chen, Y-H. (2005) 'Mechanical systems under servo constraints: the Lagrange's approach', Mechatronics, Vol. 15, No. 3, pp.317-337.

Dal Poggetto, V.F. and Serpa, A.L. (2016) 'Vehicle rollover avoidance by application of gain-scheduled LQR controllers using state observers', Vehicle System Dynamics, Vol. 54, No. 2, pp.191-209.

Du, H., Nong, Z. and Dong, G. (2010) 'Stabilizing vehicle lateral dynamics with considerations of parameter uncertainties and control saturation through robust yaw control', IEEE Transactions on Vehicular Technology, Vol. 59, No. 5, pp.2593-2597.

Edwards, C. and Spurgeon, S. (1998) Sliding Mode Control: Theory and Applications, CRC Press, Boca Raton, FL, USA.

Gáspár, P. and Bokor, J. (2006) 'A fault-tolerant rollover prevention system based on an LPV method', International Journal of Vehicle Design, Vol. 42, Nos. 3-4, pp.392-412.

Gáspár, P., Szabó, Z. and Bokor, J. (2005) 'Brake control combined with prediction to prevent the rollover of heavy vehicles', IFAC Proceedings Volumes, Vol. 38, No. 1, pp.248-253.

Hegazy, S. and Sandu, C. (2014) 'Experimental analysis and control development for vehicle rollover prevention', International Journal of Vehicle Design, Vol. 64, Nos. 2-4, pp.347-371.

Huang, H-H. (2009) Controller Design for Stability and Rollover Prevention of Multi-Body Ground Vehicles with Uncertain Dynamics and Faults, PhD Thesis, The Ohio State University.

Huang, H-H., Yedavalli, R.K. and Guenther, D.A. (2012) 'Active roll control for rollover prevention of heavy articulated vehicles with multiple-rollover-index minimisation', Vehicle System Dynamics, Vol. 50, No. 3, pp.471-493.

Imine, H., Fridman, L.M. and Madani, T. (2012) 'Steering control for rollover avoidance of heavy vehicles', IEEE Transactions on Vehicular Technology, Vol. 61, No. 8, pp.3499-3509.

Koganti, P.B. and Udwadia, F.E. (2016) 'Unified approach to modeling and control of rigid multibody systems', Journal of Guidance, Control, and Dynamics, Vol. 39, No. 12, pp.1-16.

Kunnappillil Madhusudhanan, A., Corno, M. and Holweg, E. (2015) 'Sliding mode-based lateral vehicle dynamics control using tyre force measurements', Vehicle System Dynamics, Vol. 53, No. 11, pp.1599-1619.

Larish, C., Piyabongkarn, D., Tsourapas, V. and Rajamani, R. (2013) 'A new predictive lateral load transfer ratio for rollover prevention systems', IEEE Transactions on Vehicular Technology, Vol. 62, No. 7, pp.2928-2936.

Limroth, J. (2009) Real-Time Vehicle Parameter Estimation and Adaptive Stability Control, $\mathrm{PhD}$ Thesis, Clemson University.

Odenthal, D., Bunte, T. and Ackermann, J. (1999) 'Nonlinear steering and braking control for vehicle rollover avoidance', European Control Conference (ECC), IEEE, Karlsruhe, Germany, pp.598-603.

Rajamani, R. (2011) Vehicle Dynamics and Control, Springer Science and Business Media, Berlin, Germany. 
Rajamani, R. and Piyabongkarn, D.N. (2013) 'New paradigms for the integration of yaw stability and rollover prevention functions in vehicle stability control', IEEE Transactions on Intelligent Transportation Systems, Vol. 14, No. 1, pp.249-261.

Sampson, D.J. and Cebon, D. (2003a) 'Achievable roll stability of heavy road vehicles', Proceedings of the Institution of Mechanical Engineers, Part D: Journal of Automobile Engineering, Vol. 217, No. 4, pp.269-287.

Sampson, D.J. and Cebon, D. (2003b) 'Active roll control of single unit heavy road vehicles', Vehicle System Dynamics, Vol. 40, No. 4, pp.229-270.

Shao, K., Zheng, J. and Huang, K. (2019) 'Robust active steering control for vehicle rollover prevention', International Journal of Modelling Identification and Control, Vol. 32, No. 1, pp.70-84.

Slotine, J-J.E. and Li, W. (1991) Applied Nonlinear Control, Prentice Hall, Englewood Cliffs, NJ.

Solmaz, S., Corless, M. and Shorten, R. (2007a) 'A methodology for the design of robust rollover prevention controllers for automotive vehicles: Part 2-active steering', American Control Conference(ACC), New York, NY, IEEE, pp.1606-1611.

Solmaz, S., Corless, M. and Shorten, R. (2007b) 'A methodology for the design of robust rollover prevention controllers for automotive vehicles with active steering', International Journal of Control, Vol. 80, No. 11, pp.1763-1779.

Udwadia, F.E. (2003) 'A new perspective on the tracking control of nonlinear structural and mechanical systems', Vol. 459, No. 2035, pp.1783-1800.

Udwadia, F.E. and Kalaba, R.E. (2007) Analytical Dynamics: A New Approach, Cambridge University Press, Cambridge, UK.

Udwadia, F.E. and Wanichanon, T. (2014) 'Control of uncertain nonlinear multibody mechanical systems', Journal of Applied Mechanics, Vol. 81, No. 4, pp.041020.

Yih, P. and Gerdes, J.C. (2005) 'Modification of vehicle handling characteristics via steer-by-wire', IEEE Transactions on Control Systems Technology, Vol. 13, No. 6, pp.965-976.

Yim, S. (2011) 'Design of a preview controller for vehicle rollover prevention', IEEE Transactions on Vehicular Technology, Vol. 60, No. 9, pp.4217-4226.

Zheng, J., Hai, W., Man, Z., Jin, J. and Fu, M. (2015) 'Robust motion control of a linear motor positioner using fast nonsingular terminal sliding mode', IEEE/ASME Transactions on Mechatronics, Vol. 20, No. 4, pp.1743-1752.

Zhou, J. (2009) Active Safety Measures for Vehicles Involved in Light Vehicle-to-Vehicle Impacts, PhD thesis, University of Michigan. 